\title{
Discovery and Nanosized Preparations of $(S, R)$-Tylophorine Malate as Novel anti-SARS-CoV-2 Agents
}

\author{
Ziwen Wang," Fei Ye, ${ }^{\#}$ Yue Feng, Wen Xiao, Hongiian Song, * Li Zhao, Roujian Lu, Baoying Huang, \\ Yuxiu Liu, Wenling Wang, Yongqiang Li, Yi Ding, Yanlong Zheng, Xiangrong Song,* Wenjie Tan,* \\ and Qingmin Wang*
}

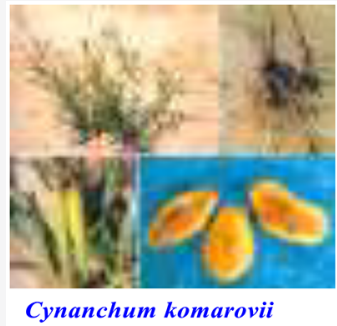

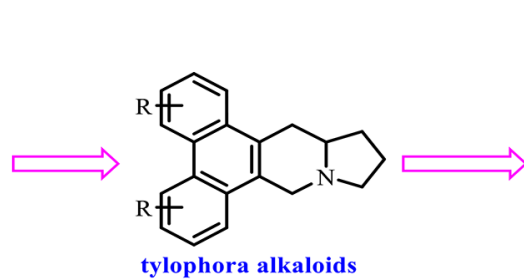

tylophora alkaloids

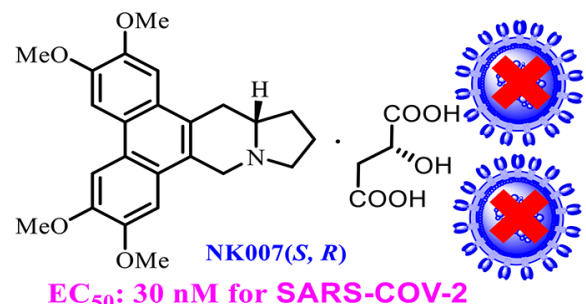

$\mathrm{EC}_{50}: 30 \mathrm{nM}$ for SARS-COV-2

Novel anti-SARS-COV-2 agent discovery based on Chinese herbal medicine

ABSTRACT: The coronavirus disease 2019 (COVID-19) pandemic caused by severe acute respiratory syndrome coronavirus 2 (SARS-CoV-2) has stimulated the search for effective drugs for its prevention and treatment. Natural products are an important source for new drug discovery. Here, we report that, $\operatorname{NK} 007(S, R)$, a tylophorine malate, displays high antiviral activity against SARS$\mathrm{CoV}-2$ with an $\mathrm{EC}_{50} 0.03 \mu \mathrm{M}$ in vitro, which is substantially lower than that of remdesivir $\left(\mathrm{EC}_{50}: 0.8 \mu \mathrm{M}\right.$ in vitro), the only authorized drug to date. The histopathological research revealed that $\mathrm{NK} 007(S, R)(5 \mathrm{mg} / \mathrm{kg} /$ dose $)$ displayed a protection effect in lung injury induced by SARS-CoV-2, which is better than remdesivir $(25 \mathrm{mg} / \mathrm{kg} / \mathrm{dose})$. We also prepared two nanosized preparations of $\mathrm{NK} 007(S, R)$, which also showed good efficacy $\left(\mathrm{EC}_{50}\right.$ : NP-NK007, $0.007 \mu \mathrm{M}$ in vitro; LP-NK007, $0.014 \mu \mathrm{M}$ in vitro). Our findings suggest that tylophora alkaloids, isolated from the traditional Chinese medicine Cynanchum komarovii AL, offer a new skeleton for the development of anticoronavirus drug candidate.

KEYWORDS: Coronavirus, COVID-19, SARS-CoV-2, Traditional Chinese medicine, Tylophorine, Nanosized preparation

\begin{abstract}
$\Delta \mathrm{s}$ of September 1, 2021, there have been 217558771 1 reported cases of coronavirus disease 2019 (COVID-19), which is caused by severe acute respiratory syndrome coronavirus 2 (SARS-CoV-2), and the disease has led to 4517240 deaths in more than 200 countries. ${ }^{1,2}$ Kissler and coworkers reported that one-off blocking measures cannot prevent the spread of SARS-CoV-2, and the United States may need to continue social isolation measures until $2022{ }^{3}$ So far, there are currently no specific drugs or vaccines available for the treatment of SARS-CoV-2 infected patients, the only authorized drug by the United States Food and Drug Administration is the nucleotide analogue remdesivir. ${ }^{4,5}$ Related studies show that remdesivir has a strong resistance to SARS-CoV-2 in vitro. ${ }^{6}$ The COVID-19 pandemic has stimulated the search for effective drugs for prevention and treatment of the disease.

The response to a viral pandemic requires the simultaneous implementation of short-term measures and long-term planning. The use of existing drugs is the focus of short-term measures, and indeed a series of first-line drugs that have been found to have potential utility for treatment of COVID-19 are
\end{abstract}

currently being explored, including ritonavir and lopinavir, ${ }^{7,8}$ ribavirin, ${ }^{9}$ chloroquine and hydroxychloroquine, ${ }^{10}$ and favipiravir and remdesivir. ${ }^{11}$ Long-term planning focuses on the identification of druggable targets and the discovery and development of new drug molecules. Progress toward targetbased design of new drugs against COVID-19 will undoubtedly be facilitated by the recent determination of the structures of the main protease $\left(\mathrm{M}^{\text {pro }}\right)^{12}$ and the RNA-dependent RNA polymerase $^{13}$ from SARS-CoV-2. The advantages of targetbased drug design are the clear target and mechanism and high selectivity, but this approach is subject to the development of drug resistance.

Received: September 2, 2021

Accepted: October 21, 2021

Published: October 24, 2021 
An alternative approach is to design new drugs based on natural products, which are excellent sources of molecules with novel chemical structures and unique mechanisms of action. Natural products often act on multiple targets, which tends to hinder the development of drug resistance. However, this approach is relatively labor-intensive, and the determining the mechanism of action of natural products can be difficult and time-consuming. Nevertheless, they have been an important source of new drugs ${ }^{14-16}$ and may be useful for the identification of molecules with activity against SARS-CoV-2.

SARS-CoV-2 is an enveloped single-stranded RNA virus. The spike protein on the viral envelope binds to the ACE2 receptors of host cells, and a viral protease can assist virus invasion. After the virus enters the cell, its genes are released, the viral replicase and transcriptase are synthesized, and RNA replication and transcription are accomplished by an RNAdependent RNA polymerase. Then structural proteins are synthesized, and finally new virus particles are assembled and released. All these steps of the virus life cycle are potential targets for drug therapy, ${ }^{6,17}$ and a number of small-molecule drugs have been shown to inhibit various viral processes at micromolar concentrations. ${ }^{12,18}$

Traditional Chinese medicines are a potential source of therapeutic small molecules, and the use of such medicines for the treatment of COVID-19 patients has recently been reviewed. ${ }^{19}$ One important traditional Chinese medicine is Cynanchum komarovii $A L$, which has been used to treat fever, diarrhea, and cholecystitis. ${ }^{20}$ This herbaceous plant, which is widely distributed in arid sandy areas of northwest China, belongs to the genus of Cynanchum Linn. in the family Asclepiadaceae. $^{21}$

In work aimed at generating active lead compounds based on traditional Chinese medicines, we discovered that Cynanchum komarovii $A L$ has good antiviral activity against tobacco mosaic virus (TMV) and that the active components were tylophora alkaloids, such as tylophorine. ${ }^{22}$ However, the tylophorine content of the plants is very low, and the molecule is light-sensitive and poorly soluble. In efforts to overcome these disadvantages, we synthesized various tylophora alkaloids (Figure 1), ${ }^{23}$ and we designed and synthesized a series of

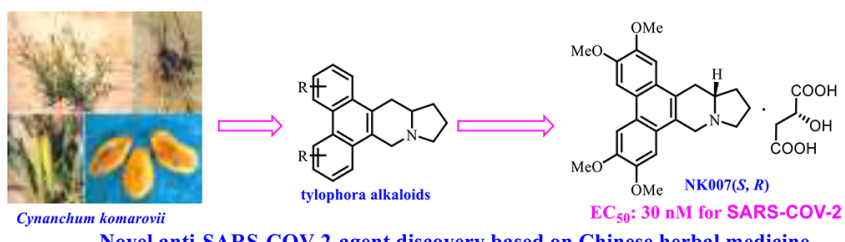

Figure 1. Discovery of NK007(S,R).

derivatives and determined their anti-TMV activities. ${ }^{24-27}$ In addition, our evaluation of the bioactivity spectrum of these compounds showed that they have good anticancer, ${ }^{28}$ anti$\mathrm{HIV}^{29}$ and immunomodulatory activities. ${ }^{30,31}$ As an optimized compound, a racemate of tylophorine malate (designated NK007) is being industrialized as an anti-TMV candidate. Herein, we report that we have prepared optically pure $\operatorname{NK} 007(S, R)$ (Figure 1) and evaluated its ability to inhibit SARS-CoV-2.

NK007 $(S, R)$ was synthesized from $S$-tylophorine ${ }^{24}$ in $97 \%$ yield (Scheme 1), and its in vitro activity against SARS-CoV-2 was tested by means of a previously reported method ${ }^{10}$ with remdesivir as a control. Under our test conditions, the $\mathrm{EC}_{50}$ of
Scheme 1. Synthesis of NK007(S,R)<smiles>COc1cc2c3c(c4cc(OC)c(OC)cc4c2cc1OC)CN1CCCC1C3</smiles>

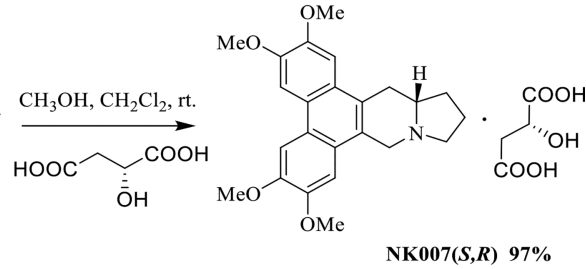

remdesivir is $0.8 \mu \mathrm{M}$ (Figure $2 \mathrm{~A}$ ), whereas that of $\mathrm{NK} 007(S, R)$ is substantially lower at $0.03 \mu \mathrm{M}$ (Figure $2 \mathrm{~B}$ ). Furthermore,

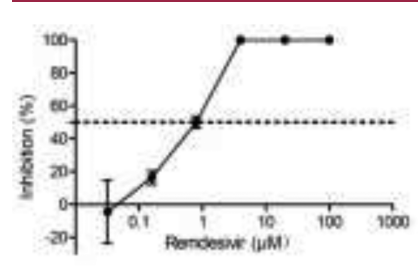

A.

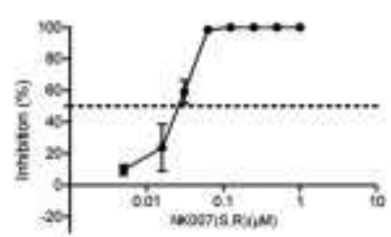

B
Figure 2. Determination of the $\mathrm{EC}_{50}$ values of remdesivir and $\operatorname{NK007}(S, R)$.

safety tests showed that $\mathrm{NK007}(S, R)$ is safe in the test state $\left(\mathrm{CC}_{50}: 26.24 \mu \mathrm{M}\right)$; the safety index is 868 (Figure 3 ).

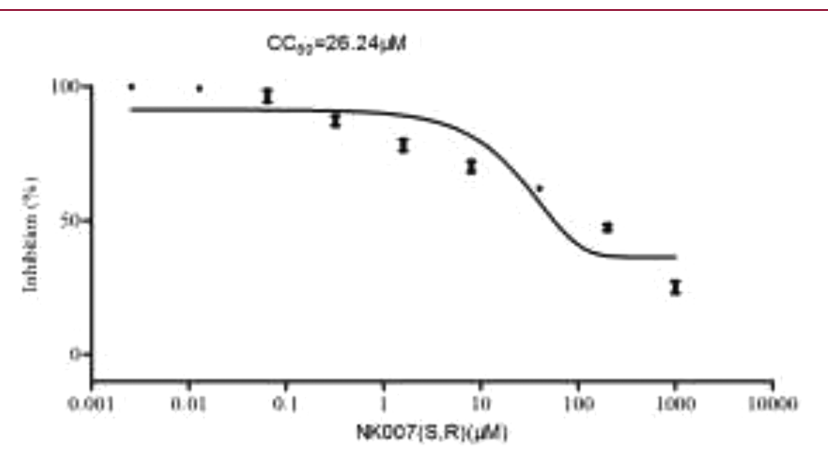

Figure 3. Determination of the $\mathrm{CC}_{50}$ value of $\mathrm{NK} 007(S, R)$.

$\mathrm{NK} 007(S, R)$ was further used to study the therapy outcome in the COVID-19 rat model. A COVID-19 golden hamster rat model was generated according to a recently published paper. $^{32}$ Briefly, golden hamster rats were randomly divided into four groups. Rats were then infected intranasally with SARS-CoV-2 $\left(1 \times 10^{5}\right.$ PFU $)$. One hour after SARS-CoV-2 infection, rats in the NS (normal saline, $0.9 \% \mathrm{NaCl}$ ) group, the remdesivir group, and the $\mathrm{NK} 007(S, R)$ group received normal saline, remdesivir, or $\operatorname{NK007}(S, R)$ intranasally. The Mock group is mock-infected rats. After virus infection at day 0 , rats received $\mathrm{NK} 007(S, R)$ or remdesivir or NS treatment 3 times as shown in Figure 4A. All rats were euthanized at day 4, and several parameters were measured, including body weight loss, SARS-CoV-2 RNA copies in the lungs, and pathological change in lung tissues.

NS-treated COVID-19 hamsters had a significant body weight loss compared with Mock hamsters on day 4 post infection ( $p$ value $=0.0093$, while $\operatorname{NK007}(S, R)$ or remdesivir treated hamsters were with no significant body weight loss when compared to the Mock hamsters (Figure 4B). We found that the average number of viral RNA copies in the lungs of $\operatorname{NK007}(S, R)\left(\sim 10^{5.7}\right)$ or remdesivir $\left(\sim 10^{5.4}\right)$ treated rats have 
A

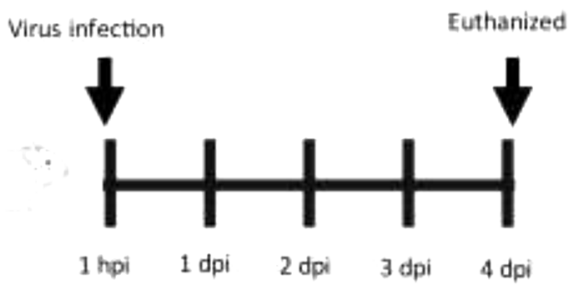

B

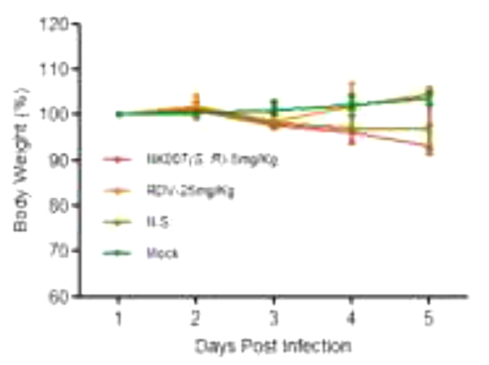

C

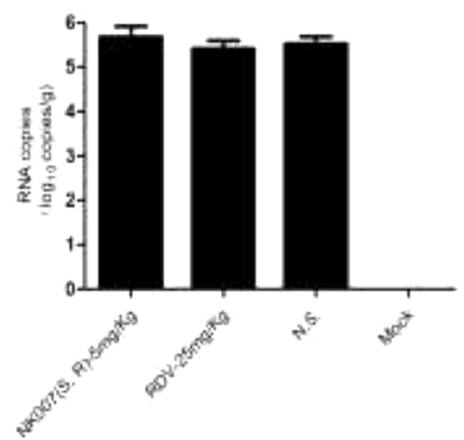

D

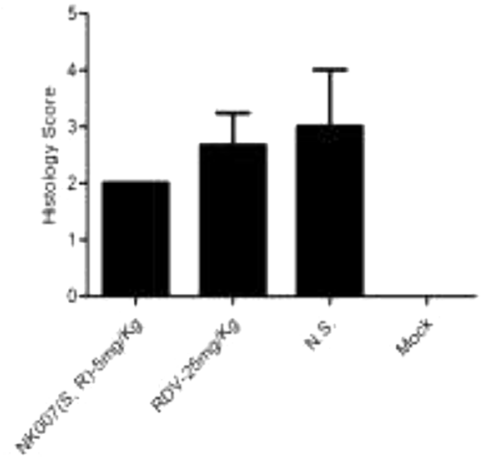

E

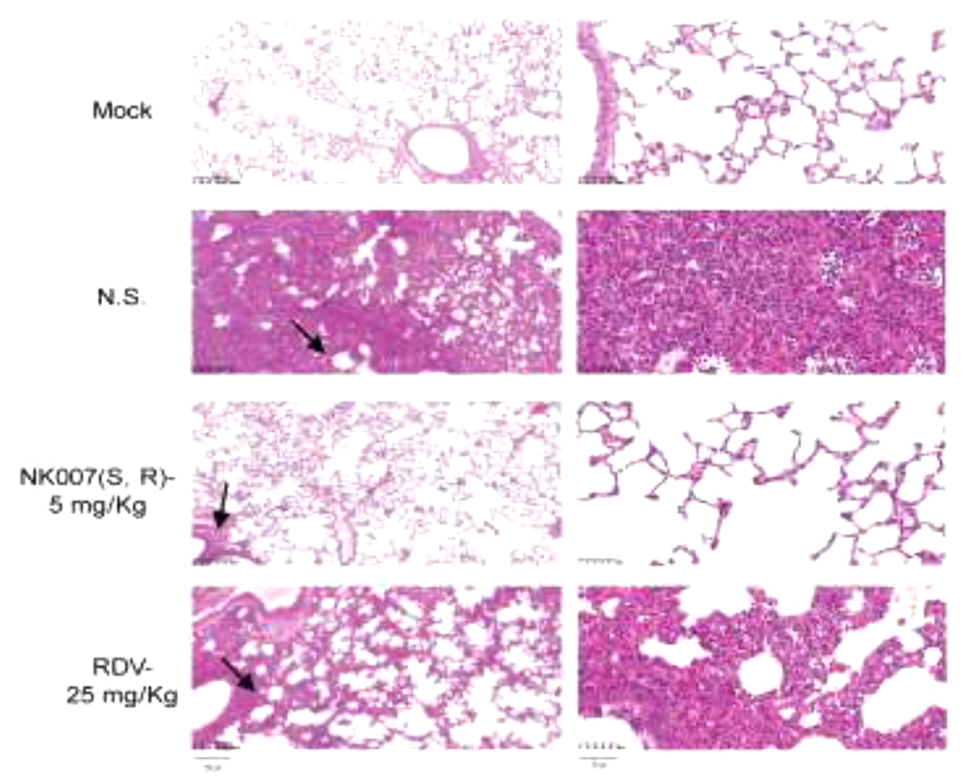

Figure 4. NK007 $(S, R)$ and remdesivir (RDV) inhibit virus replication in lungs of COVID-19 hamster rats. (A) Schematic diagram of NK007(S,R) or remdesivir treated COVID-19 hamster rats. (B) Weight loss of infected rats treated with NK007 or remdesivir. (C) Viral RNA copies in rat lungs at day 4 (unpaired $t$ test). (D) Histological scores of lung inflammation of NK007(S,R) or remdesivir treated SARS-CoV-2 infected rats (unpaired $t$ test). (E) Representative hematoxylin-eosin (HE) staining of lungs from rats harvested at day 4. Bar: $100 \mu \mathrm{m}$.

no significant difference with NS-treated infected rats $\left(\sim 10^{5.5}\right)$ (Figure $4 \mathrm{C}$ ). And the histopathological changes in rat lung tissues are a key index to assess the therapy effects of $\operatorname{NK} 007(S, R)$ or remdesivir in COVID-19 golden hamster rats. The lungs of rats were assessed by grading the injuries in accordance with the International Harmonization of Nomenclature and Diagnostic Criteria (INHAND) scoring standard. As shown in Figure 4D,E, the average histopathological score of virus-infected rats in NS group was approximately 3, along with an infiltration of lymphocytes and neutrophils, and the alveolar septum, bronchus, and perivascular interstitium were significantly widened. For rats infected by SARS-CoV-2, treatment of remdesivir at a dose of $25 \mathrm{mg} / \mathrm{kg}$ got histopathological scores, approximately 2.7 where the alveolar septum, bronchus, and perivascular interstitium were obviously widened, along with an infiltration of some of lymphocytes and neutrophils. Treatment of $\operatorname{NK007}(S, R)$ at a dose of $5 \mathrm{mg} / \mathrm{kg}$ got pathological scores, approximately 2.0. $\operatorname{NK007}(S, R)$ treatment significantly decreased lung inflammation in SARSCoV-2 infected rats. The local alveolar septum, bronchi, and 


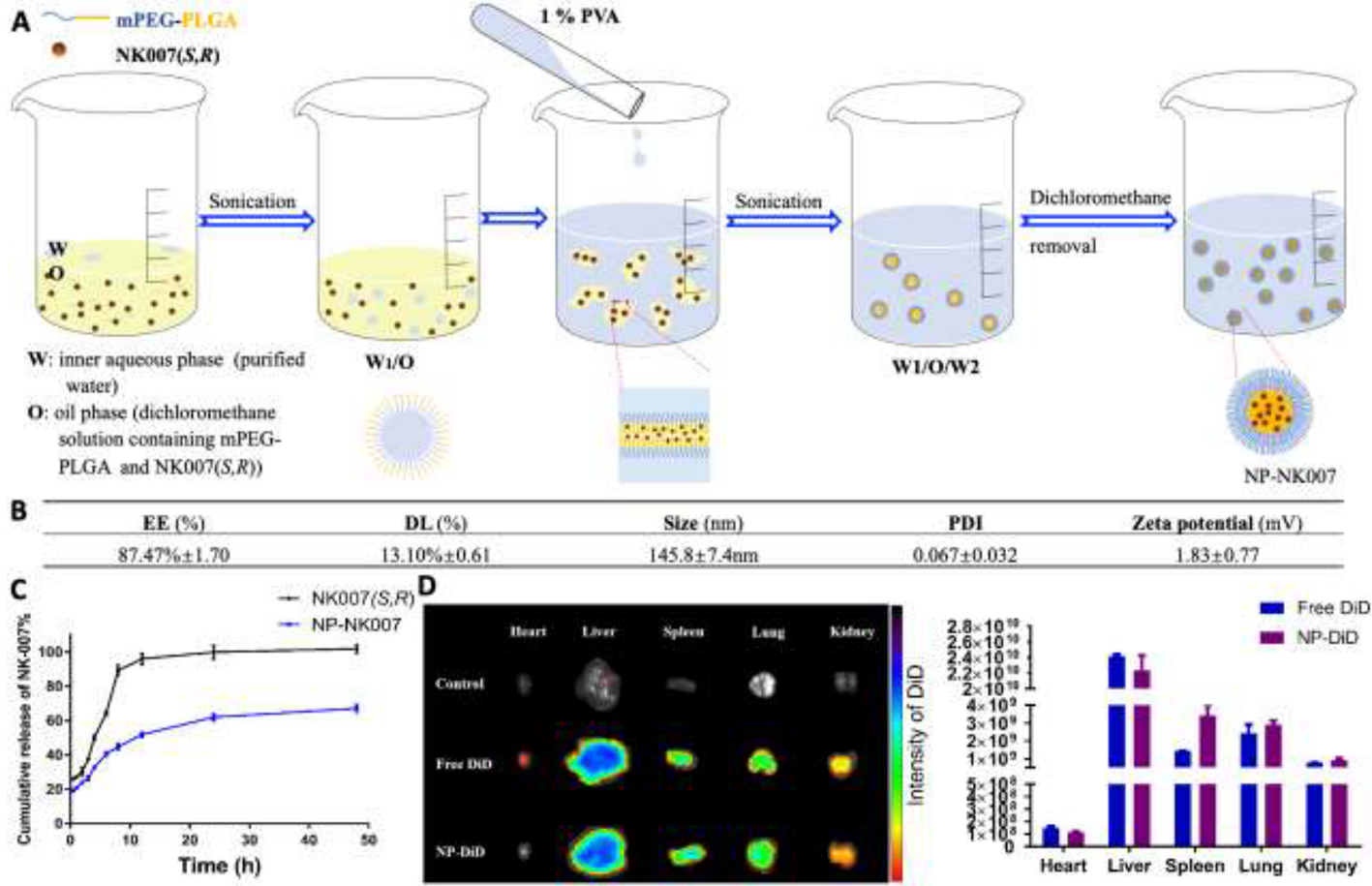

Figure 5. Preparation and characterization of NP-NK007. (A) The schematic diagram of NP-NK007 preparation processes. (B) Physical-chemical properties of NP-NK007. (C) The release profile of NK007(S,R) out of NP-NK007 in 0.5\% Tween 80 PBS phosphate buffer (pH7.4). (D) The representative fluorescent image and quantitative analysis of different vital organs at $2 \mathrm{~h}$ after intravenous injection of NP-DiD. ${ }^{*} p<0.05$, compared to the free $\mathrm{DiD}$ group.
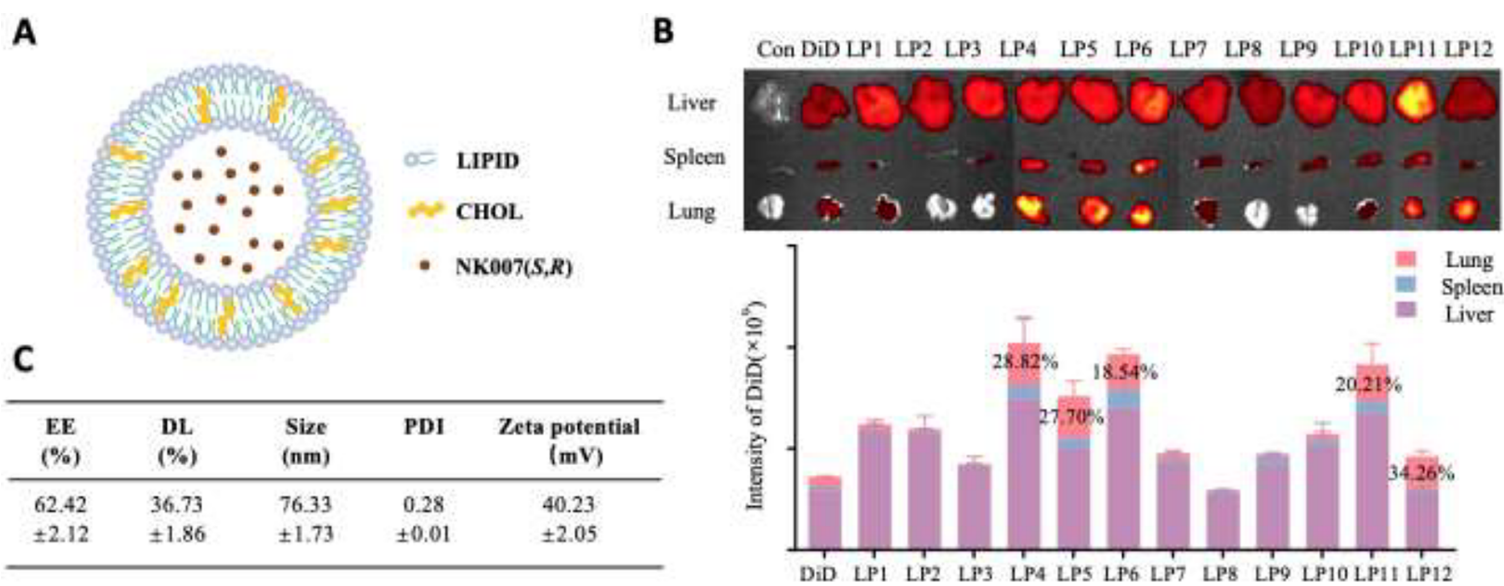

Figure 6. Structure and characterization of LP-NK007. (A) The schematic view of the LP-NK007. NK007(S,R) is encapsulated into liposomes. (B) Accumulation of LP-DiD in lungs. Representative ex vivo fluorescent images and quantitative analysis of organs at $2 \mathrm{~h}$ after intravenous injection of LP-DiD. (C) Characteristics of LP-NK007.

perivascular interstitial widening were significantly decreased in the remdesivir treatment group compared with NS-treated COVID-19 hamsters. And the $\operatorname{NK007}(S, R)$ treated group showed the lowest lung inflammation. According to histopathological results, $\mathrm{NK} 007(S, R)$ shows protection from lung injury induced by SARS-CoV-2 inflammation, and the therapy outcome of $\operatorname{NK007}(S, R)$ at a dose of $5 \mathrm{mg} / \mathrm{kg}$ is better than that of remdesivir at a dose of $25 \mathrm{mg} / \mathrm{kg}$.

The good pharmacological activity of $\operatorname{NK007}(S, R)$ lays a foundation for its application in the treatment of COVID-19. We further developed two nanosized preparations of NK007$(S, R)$ and evaluated their anti-SARS-CoV-2 activities in vitro.

The self-assembled PEG-PLGA nanoparticle loaded with NK007(S,R) (NP-NK007) was prepared using a double- emulsion (W1/O/W2) solvent evaporation method (Figure $5 \mathrm{~A}){ }^{33}$ The mean particle size of NP-NK007 was $145.8 \pm 7.4$ $\mathrm{nm}$ with a PDI of $0.067 \pm 0.032$ (Figure 5B) and a slightly positive zeta potential of $1.83 \pm 0.77 \mathrm{mV}$ (Figure S4). The encapsulation efficiency and drug loading of nanoparticles was $87.47 \% \pm 1.70$ and $13.10 \% \pm 0.61(n=3)$, respectively. The release profile of $\mathrm{NK007}(S, R)$ out of NP-NK007 was performed (Figure 5C). NP-NK007 released NK-007(S,R) gradually. Within a $48 \mathrm{~h}$ period, only $66.51 \% \pm 2.00 \%$ of NK$007(S, R)$ was released from NP-NK007. All the results illustrated that $\operatorname{NK} 007(S, R)$ was efficiently entrapped in the nanoparticles and might achieve a sustained release in vivo. The biodistribution of nanoparticles was studied in healthy C57BL/ 6 mice. As shown in Figure 5D, NP-DiD, the self-assembled 
PEG-PLGA nanoparticle loaded with a lipophilic fluorescent dye $\mathrm{DiD}$ prepared in the same method of NP-NK007, displayed enhanced distribution in the lung compared to the free $\mathrm{DiD}$. It indicated that NP-NK007 might have the potential to achieve higher antivirus activity in vivo than NK-007 $(S, R)$ because of the improved accumulation of $\mathrm{NK}-007(S, R)$ delivered by the nanoparticles in the lung.

The liposome loaded with $\mathrm{NK007}(S, R)$ (LP-NK007) was prepared by reverse-phase evaporation method. As presented in Figure 6A, we designed another platform for lung targeted delivery of $\mathrm{NK} 007(S, R)$ encapsulating into a lipid bilayer envelope. First, the in vivo distribution test was used to screen the lipid with the best lung targeting ability. Similarly, DiD was employed as the fluorescence probe to characterize the in vivo distribution profiles of different liposomes. As seen in Figure $6 \mathrm{~B}$, the relative fluorescence intensity of LP12 (DOTAP-based LP-DiD) was the strongest in the lung and was similar to free $\mathrm{DiD}$ in the spleen and liver. This implied that $\operatorname{NK007}(S, R)$ could be enriched in the lung at most when delivered by LP12 and had the least impact on other organs. Therefore, LPNK007 containing DOTAP was selected for the subsequent experiments. The characteristics of the optimal LP-NK007 were shown in Figure 6C including EE\%, DL\%, particle size, and PDI. LP-NK007 had average diameters around $75 \mathrm{~nm}$ with narrow PDI. The encapsulation efficiency and drug loading of LP-NK007 was $62.4 \%$ and $36.7 \%$, respectively.

Under our test conditions, the in vitro $\mathrm{EC}_{50}$ values of NPNK007 and LP-NK007 were 0.007 and $0.014 \mu \mathrm{M}$ respectively, and the $\mathrm{CC}_{50}$ values of NP-NK007 and LP-NK007 were 20 and $1 \mu \mathrm{M}$ respectively, which indicated that these two nanosized preparations could be used as drug sustained-release agents.

Starting from a traditional Chinese medicine, we obtained an anticoronavirus drug candidate with a novel skeleton. The compound, a tylophorine malate designated $\operatorname{NK007}(S, R)$, showed substantially higher activity $\left(\mathrm{EC}_{50}: 0.03 \mu \mathrm{M}\right)$ against SARS-CoV-2 than remdesivir $\left(\mathrm{EC}_{50}: 0.8 \mu \mathrm{M}\right) . \operatorname{NK007}(S, R)$ showed protection in lung injury induced by SARS-CoV-2 inflammation, and the therapy outcome of $\operatorname{NK007}(S, R)$ at a dose of $5 \mathrm{mg} / \mathrm{kg}$ was better than that of remdesivir at a dose of $25 \mathrm{mg} / \mathrm{kg}$. We further developed two nanosized preparations of $\operatorname{NK007}(S, R)$ and evaluated their anti-SARS-CoV-2 activities, which indicated that these two nanosized preparations could be used as drug sustained-release agents. The findings reported herein are encouraging, and we hope they will contribute to the ongoing fight against the COVID-19 pandemic.

\section{ASSOCIATED CONTENT}

\section{SI Supporting Information}

The Supporting Information is available free of charge at https://pubs.acs.org/doi/10.1021/acsmedchemlett.1c00481.

Experimental and synthetic methods, spectral characterization of compounds, tables and figures of $\mathrm{EC}_{50}$ and $\mathrm{CC}_{50}$ values, size distribution and $\zeta$ potential graphs (PDF)

\section{AUTHOR INFORMATION}

\section{Corresponding Authors}

Hongjian Song - State Key Laboratory of Elemento-Organic Chemistry, Research Institute of Elemento-Organic Chemistry, College of Chemistry, Frontiers Science Center for New
Organic Matter, Nankai University, Tianjin 300071, China; (1) orcid.org/0000-0001-7105-1196;

Email: songhongjian@nankai.edu.cn

Xiangrong Song - Department of Critical Care Medicine, Frontiers Science Center for Disease-related Molecular Network, State Key Laboratory of Biotherapy and Cancer Center, West China Hospital, Sichuan University, Chengdu 610041, China; 이이.org/0000-0002-2853-2696; Email: songxr@scu.edu.cn

Wenjie Tan - NHC Key Laboratory of Biosafety, National Institute for Viral Disease Control \& Prevention, Chinese Center for Disease Control and Prevention, China CDC, Beijing 102206, China; Email: tanwj@ivdc.chinacdc.cn

Qingmin Wang - State Key Laboratory of Elemento-Organic Chemistry, Research Institute of Elemento-Organic Chemistry, College of Chemistry, Frontiers Science Center for New Organic Matter, Nankai University, Tianjin 300071, China; 이이.org/0000-0002-6062-3766; Email: wangqm@ nankai.edu.cn

\section{Authors}

Ziwen Wang - State Key Laboratory of Elemento-Organic Chemistry, Research Institute of Elemento-Organic Chemistry, College of Chemistry, Frontiers Science Center for New Organic Matter, Nankai University, Tianjin 300071, China; Tianjin Key Laboratory of Structure and Performance for Functional Molecules, College of Chemistry, Tianjin Normal University, Tianjin 300387, China; (1) orcid.org/00000001-9798-7021

Fei Ye - NHC Key Laboratory of Biosafety, National Institute for Viral Disease Control \& Prevention, Chinese Center for Disease Control and Prevention, China CDC, Beijing 102206, China

Yue Feng - Department of Critical Care Medicine, Frontiers Science Center for Disease-related Molecular Network, State Key Laboratory of Biotherapy and Cancer Center, West China Hospital, Sichuan University, Chengdu 610041, China

Wen Xiao - Department of Critical Care Medicine, Frontiers Science Center for Disease-related Molecular Network, State Key Laboratory of Biotherapy and Cancer Center, West China Hospital, Sichuan University, Chengdu 610041, China

Li Zhao - NHC Key Laboratory of Biosafety, National Institute for Viral Disease Control \& Prevention, Chinese Center for Disease Control and Prevention, China CDC, Beijing 102206, China

Roujian Lu - NHC Key Laboratory of Biosafety, National Institute for Viral Disease Control \& Prevention, Chinese Center for Disease Control and Prevention, China CDC, Beijing 102206, China

Baoying Huang - NHC Key Laboratory of Biosafety, National Institute for Viral Disease Control \& Prevention, Chinese Center for Disease Control and Prevention, China CDC, Beijing 102206, China

Yuxiu Liu - State Key Laboratory of Elemento-Organic Chemistry, Research Institute of Elemento-Organic Chemistry, College of Chemistry, Frontiers Science Center for New Organic Matter, Nankai University, Tianjin 300071, China;

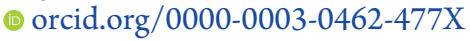

Wenling Wang - NHC Key Laboratory of Biosafety, National Institute for Viral Disease Control \& Prevention, Chinese Center for Disease Control and Prevention, China CDC, Beijing 102206, China 
Yongqiang Li - State Key Laboratory of Elemento-Organic Chemistry, Research Institute of Elemento-Organic Chemistry, College of Chemistry, Frontiers Science Center for New Organic Matter, Nankai University, Tianjin 300071, China

Yi Ding - State Key Laboratory of Elemento-Organic Chemistry, Research Institute of Elemento-Organic Chemistry, College of Chemistry, Frontiers Science Center for New Organic Matter, Nankai University, Tianjin 300071, China; Shijiazhuang Lvfeng Chemical Co., Ltd., Shijiazhuang 052560, China

Yanlong Zheng - State Key Laboratory of Elemento-Organic Chemistry, Research Institute of Elemento-Organic Chemistry, College of Chemistry, Frontiers Science Center for New Organic Matter, Nankai University, Tianjin 300071, China

Complete contact information is available at:

https://pubs.acs.org/10.1021/acsmedchemlett.1c00481

\section{Author Contributions \\ \#Ziwen Wang and Fei Ye contributed equally to this work. \\ Author Contributions}

W., W.T., and X.S. conceived the project. Z.W., H.S., Y. Liu, Y. $\mathrm{Li}$, and Y.Z. are responsible for proposal, compound design, synthesis and structure determination. F.Y., L.Z., R.L., B.H., and W.W. are responsible for the test of activity. Y.F. and W.X. are responsible for the preparation of two nanosized preparations of $\mathrm{NK} 007(S, R)$. Y.D. is responsible for providing sample.

\section{Funding}

This study was supported by Natural Science Fund of China (21732002, 21772145) and Frontiers Science Center for New Organic Matter, Nankai University (63181206). Dedicated to the 100th anniversary of Chemistry at Nankai University.

\section{Notes}

The authors declare no competing financial interest.

\section{ABBREVIATIONS}

COVID-19, the coronavirus disease 2019; SARS-CoV-2, severe acute respiratory syndrome coronavirus; ACE2, angiotensinconverting enzyme 2; INHAND, International Harmonization of Nomenclature and Diagnostic Criteria

\section{REFERENCES}

(1) World Health Organization. WHO Coronavirus (COVID-19) Dashboard. https://covid19.who.int/, 2021.

(2) Burki, T. Coronavirus in China. Lancet Respir. Med. 2020, 8, 238. (3) Kissler, S.; Tedijanto, C.; Goldstein, E.; Grad, Y.; Lipsitch, M. Projecting the transmission dynamics of SARS-CoV-2 through the postpandemic period. Science 2020, 368, 860-868.

(4) Indari, O.; Jakhmola, S.; Manivannan, E.; Jha, H. An update on antiviral therapy against SARS-CoV-2: How far have we come? Front. Pharmacol. 2021, 12, 632-677.

(5) Coronavirus (COVID-19) Update: FDA issues emergency use authorization for potential covid-19 treatment: https://www.fda.gov/ news-events/press-announcements/coronavirus-covid-19-update-fdaissues-emergency-use-authorization-potential-covid-19-treatment.

(6) Sanders, J.; Monogue, M.; Jodlowski, T.; Cutrell, J. Pharmacologic treatments for coronavirus disease 2019 (COVID19): A review. JAMA 2020, 323, 1824-1836.

(7) Chen, N.; Zhou, M.; Dong, X.; Qu, J.; Gong, F.; Han, Y.; Qiu, Y.; Wang, J.; Liu, Y.; Wei, Y.; Xia, J.; Yu, T.; Zhang, X.; Zhang, L. Epidemiological and clinical characteristics of 99 cases of 2019 novel coronavirus pneumonia in Wuhan, China: a descriptive study. Lancet 2020, 395, 507-513.
(8) Habibzadeh, P.; Stoneman, E. The novel coronavirus: A bird's eye view. Int. J. Occup. Environ. Med. 2020, 11, 65-71.

(9) Li, G.; Clercq, E. Therapeutic options for the 2019 novel coronavirus (2019-nCoV). Nat. Rev. Drug Discovery 2020, 19, 149150 .

(10) Yao, X.; Ye, F.; Zhang, M.; Cui, C.; Huang, B.; Niu, P.; Liu, X.; Zhao, L.; Dong, E.; Song, C.; Zhan, S.; Lu, R.; Li, H.; Tan, W.; Liu, D. In vitro antiviral activity and projection of optimized dosing design of hydroxychloroquine for the treatment of severe acute respiratory syndrome coronavirus 2 (SARS-CoV-2). Clin. Infect. Dis. 2020, 71, 732-739.

(11) Wang, M.; Cao, R.; Zhang, L.; Yang, X.; Liu, J.; Xu, M.; Shi, Z.; $\mathrm{Hu}, \mathrm{Z}$.; Zhong, W.; Xiao, G. Remdesivir and chloroquine effectively inhibit the recently emerged novel coronavirus $(2019-\mathrm{nCoV})$ in vitro. Cell Res. 2020, 30, 269-271.

(12) Jin, Z.; Du, X.; Xu, Y.; Deng, Y.; Liu, M.; Zhao, Y.; Zhang, B.; Li, X.; Zhang, L.; Peng, C.; Duan, Y.; Yu, J.; Wang, L.; Yang, K.; Liu, F.; Jiang, R.; Yang, X.; You, T.; Liu, X.; Yang, X.; Bai, F.; Liu, H.; Liu, X.; Guddat, L.; Xu, W.; Xiao, G.; Qin, C.; Shi, Z.; Jiang, H.; Rao, Z.; Yang, H. Structure of Mpro from COVID-19 virus and discovery of its inhibitors. Nature 2020, 582, 289-293.

(13) Gao, Y.; Yan, L.; Huang, Y.; Liu, F.; Zhao, Y.; Cao, L.; Wang, T.; Sun, Q.; Ming, Z.; Zhang, L.; Ge, J.; Zheng, L.; Zhang, Y.; Wang, H.; Zhu, Y.; Zhu, C.; Hu, T.; Hua, T.; Zhang, B.; Yang, X.; Li, J.; Yang, H.; Liu, Z.; Xu, W.; Guddat, L.; Wang, Q.; Lou, Z.; Rao, Z. Structure of the RNA-dependent RNA polymerase from COVID-19 virus. Science 2020, 368, 779-782.

(14) Rodrigues, T.; Reker, D.; Schneider, P.; Schneider, G. Counting on natural products for drug design. Nat. Chem. 2016, 8, 531-541.

(15) Atanasov, A.; Waltenberger, B.; Pferschy-Wenzig, E.; Linder, T.; Wawrosch, C.; Uhrin, P.; Temml, V.; Wang, L.; Schwaiger, S.; Heiss, E.; Rollinger, J.; Schuster, D.; Breuss, J.; Bochkov, V.; Mihovilovic, M.; Kopp, B.; Bauer, R.; Dirsch, V.; Stuppner, H. Discovery and resupply of pharmacologically active plant-derived natural products: A review. Biotechnol. Adv. 2015, 33, 1582-1614.

(16) Wang, S.; Dong, G.; Sheng, C. Structural simplification of natural products. Chem. Rev. 2019, 119, 4180-4220.

(17) Boopathi, S.; Poma, A.; Kolandaivel, P. Novel 2019 coronavirus structure, mechanism of action, antiviral drug promises and rule out against its treatment. J. Biomol. Struct. Dyn. 2021, 39, 3409-3418.

(18) Dai, W.; Zhang, B.; Jiang, X.; Su, H.; Li, J.; Zhao, Y.; Xie, X.; Jin, Z.; Peng, J.; Liu, F.; Li, C.; Li, Y.; Bai, F.; Wang, H.; Cheng, X.; Cen, X.; Hu, S.; Yang, X.; Wang, J.; Liu, X.; Xiao, G.; Jiang, H.; Rao, Z.; Zhang, L.; Xu, Y.; Yang, H.; Liu, H. Structure-based design of antiviral drug candidates targeting the SARS-CoV-2 main protease. Science 2020, 368, 1331-1335.

(19) Yang, Y.; Islam, M.; Wang, J.; Li, Y.; Chen, X. Traditional Chinese medicine in the treatment of patients infected with 2019-new coronavirus (SARS-CoV-2): A review and perspective. Int. J. Biol. Sci. 2020, 16, 1708-1717.

(20) Jiangsu New Medical College. Dictionary of Chinese Crude Drugs; Shanghai Scientific Technologic Publisher: Shanghai, 1977; p 789.

(21) Gellert, E. Structure and synthesis of phenanthroindolizidine alkaloids and some related compounds. In The Alkaloids: Chemical and Biological Perspectives; Pelletier, S., Ed.; Academic Press: New York, 1987; pp 55-132.

(22) An, T.; Huang, R.; Yang, Z.; Zhang, D.; Li, G.; Yao, Y.; Gao, J. Alkaloids from Cynanchum komarovii with inhibitory activity against the tobacco mosaic virus. Phytochemistry 2001, 58, 1267-1269.

(23) Wang, Z.; Li, Z.; Wang, K.; Wang, Q. Efficient and chirally specific synthesis of phenanthro-indolizidine alkaloids by ParhamType cycloacylation. Eur. J. Org. Chem. 2010, 2010, 292-299.

(24) Wang, K.; Su, B.; Wang, Z.; Wu, M.; Li, Z.; Hu, Y.; Fan, Z.; Mi, N.; Wang, Q. Synthesis and antiviral activities of phenanthroindolizidine alkaloids and their derivatives. J. Agric. Food Chem. 2010, 58, 2703-2709. 
(25) Wang, Z.; Wang, L.; Ma, S.; Liu, Y.; Wang, L.; Wang, Q. Design, synthesis, antiviral activity, and SARs of 14-aminophenanthroindolizidines. J. Agric. Food Chem. 2012, 60, 5825-5831.

(26) Wang, Z.; Wei, P.; Xu, X.; Liu, Y.; Wang, L.; Wang, Q. Design, synthesis, and antiviral activity evaluation of phenanthrene-based antofine derivatives. J. Agric. Food Chem. 2012, 60, 8544-8551.

(27) Wang, Z.; Wei, P.; Liu, Y.; Wang, Q. D and E rings may not be indispensable for antofine: discovery of phenanthrene and alkylamine chain containing antofine derivatives as novel antiviral agents against tobacco mosaic virus (TMV) based on interaction of antofine and TMV RNA. J. Agric. Food Chem. 2014, 62, 10393-10404.

(28) Wang, Z.; Wu, M.; Wang, Y.; Li, Z.; Wang, L.; Han, G.; Chen, F.; Liu, Y.; Wang, K.; Zhang, A.; Meng, L.; Wang, Q. Synthesis and SAR studies of phenanthroindolizidine and phenanthroquinolizidine alkaloids as potent antitumor agents. Eur. J. Med. Chem. 2012, 51, 250-258.

(29) Wang, Z.; Wang, M.; Wang, L.; Yao, X.; Li, Y.; Tan, J.; Qiao, W.; Geng, Y.; Liu, Y.; Wang, Q. First discovery of tylophora alkaloids as HIV inhibitors. Lett. Drug Des. Discovery 2015, 12, 277-283.

(30) Wen, T.; Li, Y.; Wu, M.; Sun, X.; Bao, X.; Lin, Y.; Hao, J.; Han, L.; Cao, G.; Wang, Z.; Liu, Y.; Wu, Z.; Hong, Z.; Wang, P.; Zhao, L.; Li, Z.; Wang, Q.; Yin, Z. Therapeutic effects of a novel tylophorine analog, NK-007, on collagen-induced arthritis through suppressing tumor necrosis factor $\alpha$ production and Th17 cell differentiation. Arthritis Rheum. 2012, 64, 2896-2906.

(31) Wen, T.; Li, Y.; Wu, M.; Chen, X.; Han, L.; Bao, X.; Wang, Z.; Wang, K.; Hu, Y.; Zhou, X.; Wu, Z.; Wang, P.; Hong, Z.; Zhao, L.; Wang, Q.; Yin, Z. A novel tylophorine analog NK-007 ameliorates colitis through inhibition of innate immune response. Int. Immunopharmacol. 2012, 14, 487-494.

(32) Sia, S.; Yan, L.; Chin, A.; Fung, K.; Choy, K.; Wong, A.; Kaewpreedee, P.; Perera, R.; Poon, L.; Nicholls, J.; Peiris, M.; Yen, H. Pathogenesis and transmission of SARS-CoV-2 in golden hamsters. Nature 2020, 583, 834-838.

(33) Zhao, S.; Li, J.; Zhou, Y.; Huang, L.; Li, Y.; Xu, J.; Fu, C.; Guo, X.; Yang, J. Lipid nanoparticles-encapsulated YF4: A potential therapeutic oral peptide delivery system for hypertension treatment. Front. Pharmacol. 2019, 10, 102. 